\title{
Nature Index tables
}

The Index's leading countries and institutions for high-quality science, ordered by weighted fractional count (WFC) for 2014. Also shown are the total number of articles, and the change in WFC from 2013. Articles are from the 68 natural science journals that comprise the Nature Index (see Guide to the Nature Index, page S30).

\section{TOP 100 COUNTRIES}

\begin{tabular}{|c|c|c|c|c|c|}
\hline 2014 & COUNTRY & WFC & $\begin{array}{l}\text { ARTICLE } \\
\text { COUNT }\end{array}$ & $\begin{array}{l}2013 \\
\text { WFC }\end{array}$ & $\begin{array}{l}\text { 2013-2014 } \\
\text { CHANGE IN WFC }\end{array}$ \\
\hline 1 & United States & $17,936.51$ & 26,638 & $18,581.10$ & $-3.5 \%$ \\
\hline 2 & China & $6,037.22$ & 8,641 & $5,204.06$ & $16.0 \%$ \\
\hline 3 & Germany & $4,018.73$ & 8,582 & $4,073.53$ & $-1.3 \%$ \\
\hline 4 & United Kingdom & $3,249.72$ & 7,592 & $3,271.65$ & $-0.7 \%$ \\
\hline 5 & Japan & $3,200.43$ & 4,976 & $3,365.50$ & $-4.9 \%$ \\
\hline 6 & France & $2,221.95$ & 5,243 & $2,232.59$ & $-0.5 \%$ \\
\hline 7 & Canada & $1,488.90$ & 3,226 & $1,480.64$ & $0.6 \%$ \\
\hline 8 & Switzerland & $1,293.75$ & 2,715 & $1,169.17$ & $10.7 \%$ \\
\hline 9 & South Korea & $1,167.66$ & 1,969 & $1,150.07$ & $1.5 \%$ \\
\hline 10 & Spain & $1,090.72$ & 2,897 & $1,177.00$ & $-7.3 \%$ \\
\hline 11 & Italy & $1,046.98$ & 3,052 & $1,078.08$ & $-2.9 \%$ \\
\hline 12 & Australia & 951.22 & 2,497 & 932.86 & $2.0 \%$ \\
\hline 13 & India & 921.77 & 1,484 & 850.97 & $8.3 \%$ \\
\hline 14 & Netherlands & 757.33 & 2,189 & 759.47 & $-0.3 \%$ \\
\hline 15 & Singapore & 520.62 & 873 & 483.20 & $7.7 \%$ \\
\hline 16 & Sweden & 514.55 & 1,407 & 496.38 & $3.7 \%$ \\
\hline 17 & Israel & 492.44 & 1,012 & 473.15 & $4.1 \%$ \\
\hline 18 & Taiwan & 481.47 & 888 & 543.18 & $-11.4 \%$ \\
\hline 19 & Russia & 370.18 & 1,147 & 344.26 & $7.5 \%$ \\
\hline 20 & Belgium & 348.91 & 1,082 & 327.23 & $6.6 \%$ \\
\hline 21 & Denmark & 321.26 & 1,036 & 296.85 & $8.2 \%$ \\
\hline 22 & Austria & 318.98 & 856 & 279.97 & $13.9 \%$ \\
\hline 23 & Brazil & 237.01 & 715 & 233.69 & $1.4 \%$ \\
\hline 24 & Poland & 209.69 & 686 & 216.55 & $-3.2 \%$ \\
\hline 25 & Finland & 194.97 & 590 & 191.87 & $1.6 \%$ \\
\hline 26 & Norway & 149.81 & 415 & 122.42 & $22.4 \%$ \\
\hline 27 & Czech Republic & 126.33 & 372 & 118.43 & $6.7 \%$ \\
\hline 28 & Portugal & 117.45 & 416 & 123.53 & $-4.9 \%$ \\
\hline 29 & Ireland & 113.91 & 331 & 116.73 & $-2.4 \%$ \\
\hline 30 & Argentina & 98.58 & 321 & 105.55 & $-6.6 \%$ \\
\hline 31 & New Zealand & 95.69 & 275 & 117.58 & $-18.6 \%$ \\
\hline 32 & Chile & 95.13 & 857 & 75.43 & $26.1 \%$ \\
\hline 33 & Greece & 91.77 & 320 & 90.15 & $1.8 \%$ \\
\hline 34 & Mexico & 84.60 & 391 & 77.08 & $9.7 \%$ \\
\hline 35 & South Africa & 81.67 & 418 & 81.62 & $0.1 \%$ \\
\hline 36 & Turkey & 79.24 & 237 & 59.07 & $34.1 \%$ \\
\hline 37 & Hungary & 78.52 & 278 & 54.92 & $43.0 \%$ \\
\hline 38 & Saudi Arabia & 76.63 & 384 & 76.64 & $0.0 \%$ \\
\hline 39 & Iran & 67.87 & 147 & 58.55 & $15.9 \%$ \\
\hline 40 & Ukraine & 37.06 & 173 & 28.58 & $29.7 \%$ \\
\hline 41 & Slovenia & 36.36 & 106 & 43.20 & $-15.8 \%$ \\
\hline 42 & Thailand & 22.69 & 93 & 25.69 & $-11.7 \%$ \\
\hline
\end{tabular}




\begin{tabular}{|c|c|c|c|c|c|}
\hline 2014 & COUNTRY & WFC & $\begin{array}{l}\text { ARTICLE } \\
\text { COUNT }\end{array}$ & $\begin{array}{l}2013 \\
\text { WFC }\end{array}$ & $\begin{array}{l}\text { 2013-2014 } \\
\text { CHANGE IN WFC }\end{array}$ \\
\hline 43 & Estonia & 22.31 & 92 & 13.27 & $68.1 \%$ \\
\hline 44 & Romania & 21.41 & 79 & 17.47 & $22.6 \%$ \\
\hline 45 & Croatia & 17.56 & 102 & 21.86 & $-19.7 \%$ \\
\hline 46 & Serbia & 17.00 & 55 & 13.95 & $21.9 \%$ \\
\hline 47 & Lithuania & 16.85 & 58 & 11.26 & $49.7 \%$ \\
\hline 48 & Malaysia & 15.81 & 54 & 14.14 & $11.8 \%$ \\
\hline 49 & Egypt & 14.32 & 87 & 12.04 & $18.9 \%$ \\
\hline 50 & Slovakia & 14.16 & 64 & 12.57 & $12.6 \%$ \\
\hline 51 & Puerto Rico & 13.66 & 38 & 11.24 & $21.6 \%$ \\
\hline 52 & Iceland & 12.87 & 65 & 13.90 & $-7.4 \%$ \\
\hline 53 & Pakistan & 12.83 & 42 & 18.03 & $-28.8 \%$ \\
\hline 54 & Cyprus & 10.95 & 28 & 8.90 & $23.1 \%$ \\
\hline 55 & Luxembourg & 10.94 & 35 & 10.55 & $3.7 \%$ \\
\hline 56 & Uruguay & 9.55 & 22 & 5.21 & $83.5 \%$ \\
\hline 57 & United Arab Emirates & 9.37 & 49 & 7.08 & $32.2 \%$ \\
\hline 58 & Colombia & 9.36 & 56 & 9.45 & $-1.0 \%$ \\
\hline 59 & Panama & 8.84 & 32 & 6.61 & $33.9 \%$ \\
\hline 60 & Bulgaria & 8.59 & 63 & 9.49 & $-9.5 \%$ \\
\hline 61 & Armenia & 8.38 & 30 & 7.98 & $5.0 \%$ \\
\hline 62 & Vietnam & 7.53 & 29 & 8.23 & $-8.4 \%$ \\
\hline 63 & Belarus & 6.15 & 22 & 3.95 & $55.9 \%$ \\
\hline 64 & Indonesia & 5.92 & 30 & 4.28 & $38.4 \%$ \\
\hline 65 & Kenya & 5.64 & 25 & 5.36 & $5.2 \%$ \\
\hline 66 & Qatar & 4.98 & 35 & 3.96 & $25.6 \%$ \\
\hline 67 & Peru & 4.92 & 29 & 4.22 & $16.6 \%$ \\
\hline 68 & Algeria & 4.65 & 22 & 1.10 & $322.5 \%$ \\
\hline 69 & Georgia & 4.15 & 27 & 2.45 & $69.6 \%$ \\
\hline 70 & Costa Rica & 3.50 & 25 & 1.49 & $134.6 \%$ \\
\hline 71 & Lebanon & 3.08 & 14 & 3.34 & $-7.9 \%$ \\
\hline 72 & Tunisia & 2.75 & 13 & 2.84 & $-2.8 \%$ \\
\hline 73 & Ecuador & 2.48 & 19 & 2.48 & $0.1 \%$ \\
\hline 74 & Bangladesh & 2.45 & 14 & 2.28 & $7.7 \%$ \\
\hline 75 & Venezuela & 2.40 & 27 & 1.83 & $31.2 \%$ \\
\hline 76 & Morocco & 2.37 & 18 & 3.46 & $-31.4 \%$ \\
\hline 77 & Latvia & 2.18 & 12 & 3.18 & $-31.4 \%$ \\
\hline 78 & Cuba & 2.03 & 9 & 4.86 & $-58.2 \%$ \\
\hline 79 & Oman & 2.00 & 8 & 1.29 & $54.8 \%$ \\
\hline 80 & Kazakhstan & 1.93 & 9 & 2.85 & $-32.2 \%$ \\
\hline 81 & Philippines & 1.82 & 16 & 3.33 & $-45.4 \%$ \\
\hline 82 & Moldova & 1.68 & 10 & 3.35 & $-49.8 \%$ \\
\hline 83 & Nepal & 1.64 & 8 & 0.82 & $99.4 \%$ \\
\hline 84 & Benin & 1.62 & 6 & 0.37 & $341.4 \%$ \\
\hline 85 & Uganda & 1.61 & 11 & 0.29 & $452.6 \%$ \\
\hline 86 & Mongolia & 1.37 & 5 & 0.62 & $119.1 \%$ \\
\hline 87 & Malta & 1.30 & 5 & 0.46 & $179.4 \%$ \\
\hline 88 & Senegal & 1.17 & 4 & 0.56 & $110.1 \%$ \\
\hline 89 & Cameroon & 1.16 & 8 & 0.81 & $42.9 \%$ \\
\hline 90 & Uzbekistan & 1.16 & 10 & 0.17 & $576.6 \%$ \\
\hline 91 & Bolivia & 1.05 & 9 & 0.48 & $120.2 \%$ \\
\hline 92 & Mali & 1.00 & 3 & 0.19 & $415.5 \%$ \\
\hline 93 & Macedonia & 1.00 & 6 & 0.26 & $290.5 \%$ \\
\hline 94 & Kuwait & 0.95 & 7 & 3.17 & $-70.0 \%$ \\
\hline 95 & Namibia & 0.95 & 4 & 0.09 & $988.5 \%$ \\
\hline 96 & Burkina Faso & 0.93 & 4 & 0.05 & $1,944.6 \%$ \\
\hline 97 & Azerbaijan & 0.92 & 5 & 0.56 & $63.0 \%$ \\
\hline 98 & Iraq & 0.84 & 12 & 0.74 & $13.8 \%$ \\
\hline 99 & Sri Lanka & 0.83 & 8 & 0.70 & $18.6 \%$ \\
\hline 100 & Kyrgyzstan & 0.81 & 2 & 0.81 & $-0.2 \%$ \\
\hline
\end{tabular}




\section{TOP 200 INSTITUTIONS}

\begin{tabular}{|c|c|c|c|c|c|c|}
\hline 2014 & INSTITUTION & COUNTRY & WFC & $\begin{array}{l}\text { ARTICLE } \\
\text { COUNT }\end{array}$ & $\begin{array}{l}2013 \\
\text { WFC }\end{array}$ & $\begin{array}{l}2013-2014 \\
\text { CHANGE IN WFC }\end{array}$ \\
\hline 1 & Chinese Academy of Sciences (CAS) & China & $1,307.74$ & 3,124 & $1,209.37$ & $8.1 \%$ \\
\hline 2 & Harvard University & United States & 865.09 & 2,615 & 852.85 & $1.4 \%$ \\
\hline 3 & French National Centre for Scientific Research (CNRS) & France & 761.48 & 4,907 & 719.07 & $5.9 \%$ \\
\hline 4 & Max Planck Society & Germany & 648.86 & 2,950 & 727.32 & $-10.8 \%$ \\
\hline 5 & Stanford University (SU) & United States & 538.57 & 1,265 & 550.20 & $-2.1 \%$ \\
\hline 6 & Massachusetts Institute of Technology (MIT) & United States & 501.38 & 1,483 & 507.17 & $-1.1 \%$ \\
\hline 7 & The University of Tokyo (UTokyo) & Japan & 456.23 & 1,251 & 472.88 & $-3.5 \%$ \\
\hline 8 & Helmholtz Association of German Research Centres (Helmholtz) & Germany & 440.09 & 1,629 & 422.02 & $4.3 \%$ \\
\hline 9 & University of Cambridge & United Kingdom & 403.11 & 1,384 & 401.39 & $0.4 \%$ \\
\hline 10 & University of California Berkeley (UC Berkeley) & United States & 365.55 & 1,236 & 362.16 & $0.9 \%$ \\
\hline 11 & University of Oxford & United Kingdom & 355.99 & 1,179 & 382.41 & $-6.9 \%$ \\
\hline 12 & Swiss Federal Institute of Technology Zurich (ETH Zurich) & Switzerland & 347.29 & 978 & 275.31 & $26.1 \%$ \\
\hline 13 & National Institutes of Health (NIH) & United States & 342.43 & 771 & 391.02 & $-12.4 \%$ \\
\hline 14 & University of California, San Diego (UC San Diego) & United States & 334.34 & 832 & 344.80 & $-3.0 \%$ \\
\hline 15 & Yale University & United States & 303.63 & 896 & 289.56 & $4.9 \%$ \\
\hline 16 & University of Michigan (U-M) & United States & 298.80 & 855 & 344.95 & $-13.4 \%$ \\
\hline 17 & Peking University (PKU) & China & 291.57 & 937 & 275.46 & $5.8 \%$ \\
\hline 18 & Kyoto University & Japan & 286.15 & 716 & 313.51 & $-8.7 \%$ \\
\hline 19 & The University of Texas at Austin (UT Austin) & United States & 274.17 & 629 & 246.84 & $11.1 \%$ \\
\hline 20 & California Institute of Technology (Caltech) & United States & 270.83 & 1199 & 280.77 & $-3.5 \%$ \\
\hline 21 & University of Toronto ( $\mathrm{U}$ of $\mathrm{T}$ ) & Canada & 268.61 & 829 & 256.09 & $4.9 \%$ \\
\hline 22 & University of California Los Angeles (UCLA) & United States & 260.59 & 744 & 286.64 & $-9.1 \%$ \\
\hline 23 & University of Washington (UW) & United States & 259.61 & 766 & 248.89 & $4.3 \%$ \\
\hline 24 & Northwestern University (NU) & United States & 259.33 & 586 & 298.02 & $-13.0 \%$ \\
\hline 25 & Columbia University in the City of New York (CU) & United States & 258.13 & 851 & 257.32 & $0.3 \%$ \\
\hline 26 & University of Pennsylvania (Penn) & United States & 252.97 & 589 & 295.67 & $-14.4 \%$ \\
\hline 27 & Spanish National Research Council (CSIC) & Spain & 242.90 & 1846 & 240.00 & $1.2 \%$ \\
\hline 28 & University of Wisconsin-Madison (UW Madison) & United States & 232.81 & 654 & 251.98 & $-7.6 \%$ \\
\hline 29 & Swiss Federal Institute of Technology in Lausanne (EPFL) & Switzerland & 232.70 & 687 & 207.58 & $12.1 \%$ \\
\hline 30 & The Johns Hopkins University (JHU) & United States & 226.16 & 783 & 223.91 & $1.0 \%$ \\
\hline 31 & Cornell University & United States & 226.04 & 689 & 247.53 & $-8.7 \%$ \\
\hline 32 & Osaka University & Japan & 218.77 & 555 & 219.88 & $-0.5 \%$ \\
\hline 33 & The University of Chicago (UChicago) & United States & 208.64 & 724 & 190.48 & $9.5 \%$ \\
\hline 34 & Princeton University & United States & 207.12 & 680 & 230.61 & $-10.2 \%$ \\
\hline 35 & University of Illinois at Urbana Champaign (UIUC) & United States & 206.36 & 551 & 229.86 & $-10.2 \%$ \\
\hline 36 & Tsinghua University (TH) & China & 205.58 & 611 & 195.00 & $5.4 \%$ \\
\hline 37 & Nanjing University (NJU) & China & 202.30 & 474 & 196.00 & $3.2 \%$ \\
\hline 38 & University of California Santa Barbara (UCSB) & United States & 201.06 & 552 & 196.04 & $2.6 \%$ \\
\hline 39 & Imperial College London (ICL) & United Kingdom & 200.52 & 727 & 185.01 & $8.4 \%$ \\
\hline 40 & Nanyang Technological University (NTU) & Singapore & 199.86 & 371 & 187.38 & $6.7 \%$ \\
\hline 41 & University of Science and Technology of China (USTC) & China & 191.75 & 541 & 175.73 & $9.1 \%$ \\
\hline 42 & National University of Singapore (NUS) & Singapore & 190.30 & 465 & 191.76 & $-0.8 \%$ \\
\hline 43 & Zhejiang University (ZJU) & China & 188.48 & 352 & 150.42 & $25.3 \%$ \\
\hline 44 & University of California San Francisco (UCSF) & United States & 188.02 & 446 & 221.76 & $-15.2 \%$ \\
\hline 45 & University College London (UCL) & United Kingdom & 185.66 & 799 & 183.78 & $1.0 \%$ \\
\hline 46 & Tohoku University & Japan & 178.14 & 426 & 189.32 & $-5.9 \%$ \\
\hline 47 & University of North Carolina at Chapel Hill (UNC) & United States & 177.36 & 387 & 170.63 & $3.9 \%$ \\
\hline 48 & Russian Academy of Sciences (RAS) & Russia & 171.47 & 910 & 165.96 & $3.3 \%$ \\
\hline 49 & Pennsylvania State University (Penn State) & United States & 171.14 & 587 & 185.84 & $-7.9 \%$ \\
\hline
\end{tabular}




\begin{tabular}{|c|c|c|c|c|c|c|}
\hline 2014 & INSTITUTION & COUNTRY & WFC & $\begin{array}{l}\text { ARTICLE } \\
\text { COUNT }\end{array}$ & $\begin{array}{l}2013 \\
\text { WFC }\end{array}$ & $\begin{array}{l}\text { 2013-2014 } \\
\text { CHANGE IN WFC }\end{array}$ \\
\hline 50 & University of California Davis (UC Davis) & United States & 169.69 & 478 & 183.16 & $-7.4 \%$ \\
\hline 51 & Indian Institutes of Technology (IITs) & India & 168.18 & 288 & 128.43 & $30.9 \%$ \\
\hline 52 & Duke University & United States & 167.60 & 501 & 173.44 & $-3.4 \%$ \\
\hline 53 & Fudan University & China & 162.59 & 344 & 129.22 & $25.8 \%$ \\
\hline 54 & University of Maryland, College Park (UMCP) & United States & 162.48 & 740 & 161.78 & $0.4 \%$ \\
\hline 55 & Washington University in St. Louis (WUSTL) & United States & 161.42 & 379 & 173.04 & $-6.7 \%$ \\
\hline 56 & Lawrence Berkeley National Laboratory (LBNL) & United States & 161.03 & 781 & 153.57 & $4.9 \%$ \\
\hline 57 & Seoul National University (SNU) & South Korea & 160.31 & 429 & 158.14 & $1.4 \%$ \\
\hline 58 & University of Minnesota (UMN) & United States & 158.05 & 491 & 178.08 & $-11.2 \%$ \\
\hline 59 & Ludwig Maximilian University of Munich (LMU) & Germany & 157.57 & 627 & 154.25 & $2.2 \%$ \\
\hline 60 & Rutgers, The State University of New Jersey (RU) & United States & 148.48 & 455 & 154.02 & $-3.6 \%$ \\
\hline 61 & RIKEN & Japan & 144.43 & 497 & 165.96 & $-13.0 \%$ \\
\hline 62 & New York University (NYU) & United States & 144.08 & 431 & 139.91 & $3.0 \%$ \\
\hline 63 & University of Colorado Boulder (CU-Boulder) & United States & 143.58 & 553 & 152.16 & $-5.6 \%$ \\
\hline 64 & Weizmann Institute of Science (WIS) & Israel & 142.96 & 296 & 150.96 & $-5.3 \%$ \\
\hline 65 & The Ohio State University (OSU) & United States & 142.01 & 555 & 142.91 & $-0.6 \%$ \\
\hline 66 & The University of Manchester (UoM) & United Kingdom & 141.03 & 565 & 125.06 & $12.8 \%$ \\
\hline 67 & The Scripps Research Institute (TSRI) & United States & 134.75 & 288 & 178.38 & $-24.5 \%$ \\
\hline 68 & McGill University & Canada & 133.57 & 468 & 136.55 & $-2.2 \%$ \\
\hline 69 & National Research Council (CNR) & Italy & 130.31 & 504 & 150.02 & $-13.1 \%$ \\
\hline 70 & The University of British Columbia (UBC) & Canada & 130.25 & 463 & 130.64 & $-0.3 \%$ \\
\hline 71 & University of California Irvine (UCI) & United States & 130.13 & 405 & 147.32 & $-11.7 \%$ \\
\hline 72 & Council of Scientific and Industrial Research (CSIR) & India & 128.64 & 196 & 115.24 & $11.6 \%$ \\
\hline 73 & Texas A\&M University (TAMU) & United States & 128.34 & 394 & 147.32 & $-12.9 \%$ \\
\hline 74 & Leibniz Association & Germany & 128.11 & 540 & 117.56 & $9.0 \%$ \\
\hline 75 & Tokyo Institute of Technology (Tokyo Tech) & Japan & 127.23 & 331 & 105.59 & $20.5 \%$ \\
\hline 76 & University of Bristol (UoB) & United Kingdom & 125.73 & 399 & 109.62 & $14.7 \%$ \\
\hline 77 & Nagoya University & Japan & 125.28 & 432 & 132.97 & $-5.8 \%$ \\
\hline 78 & University of Copenhagen (UCPH) & Denmark & 124.84 & 615 & 117.29 & $6.4 \%$ \\
\hline 79 & Purdue University & United States & 123.37 & 349 & 112.74 & $9.4 \%$ \\
\hline 80 & University of Pittsburgh (Pitt) & United States & 122.10 & 380 & 148.22 & $-17.6 \%$ \\
\hline 81 & Technical University Munich (TUM) & Germany & 121.39 & 477 & 125.21 & $-3.1 \%$ \\
\hline 82 & Vanderbilt University (VU) & United States & 121.38 & 368 & 101.83 & $19.2 \%$ \\
\hline 83 & The University of Texas Southwestern Medical Center (UT Southwestern Medical Center) & United States & 117.93 & 229 & 124.11 & $-5.0 \%$ \\
\hline 84 & Korea Advanced Institute of Science and Technology (KAIST) & South Korea & 116.39 & 228 & 103.03 & $13.0 \%$ \\
\hline 85 & Argonne National Laboratory (ANL) & United States & 113.69 & 475 & 113.74 & $0.0 \%$ \\
\hline 86 & Atomic Energy and Alternative Energies Commission (CEA) & France & 112.95 & 1080 & 138.71 & $-18.6 \%$ \\
\hline 87 & The University of Edinburgh & United Kingdom & 112.04 & 796 & 110.44 & $1.4 \%$ \\
\hline 88 & Indiana University (IU) & United States & 111.67 & 354 & 119.17 & $-6.3 \%$ \\
\hline 89 & University of Southern California (USC) & United States & 111.59 & 270 & 127.44 & $-12.4 \%$ \\
\hline 90 & Heidelberg University (Uni Heidelberg) & Germany & 111.39 & 631 & 105.66 & $5.4 \%$ \\
\hline 91 & University of Geneva (UNIGE) & Switzerland & 111.18 & 446 & 108.07 & $2.9 \%$ \\
\hline 92 & The University of Queensland (UQ) & Australia & 108.83 & 392 & 94.78 & $14.8 \%$ \\
\hline 93 & Georgia Institute of Technology (Georgia Tech) & United States & 108.31 & 267 & 137.43 & $-21.2 \%$ \\
\hline 94 & Emory University & United States & 107.54 & 239 & 108.36 & $-0.8 \%$ \\
\hline 95 & National Taiwan University (NTU) & Taiwan & 107.49 & 299 & 102.57 & $4.8 \%$ \\
\hline 96 & University of Florida (UF) & United States & 106.90 & 414 & 105.28 & $1.5 \%$ \\
\hline 97 & Shanghai Jiao Tong University (SJTU) & China & 106.84 & 282 & 96.01 & $11.3 \%$ \\
\hline 98 & Lanzhou University (LZU) & China & 106.31 & 176 & 69.71 & $52.5 \%$ \\
\hline 99 & University of Utah (Utah) & United States & 105.95 & 282 & 113.06 & $-6.3 \%$ \\
\hline 100 & Monash University & Australia & 103.11 & 382 & 94.89 & $8.7 \%$ \\
\hline
\end{tabular}




\begin{tabular}{|c|c|c|c|c|c|c|}
\hline 2014 & INSTITUTION & COUNTRY & WFC & $\begin{array}{l}\text { ARTICLE } \\
\text { COUNT }\end{array}$ & $\begin{array}{l}2013 \\
\text { WFC }\end{array}$ & $\begin{array}{l}2013-2014 \\
\text { CHANGE IN WFC }\end{array}$ \\
\hline 101 & Los Alamos National Laboratory (LANL) & United States & 102.26 & 375 & 115.64 & $-11.6 \%$ \\
\hline 102 & University of Münster (WWU) & Germany & 101.94 & 221 & 94.17 & $8.2 \%$ \\
\hline 103 & Jilin University (JLU) & China & 101.87 & 184 & 97.72 & $4.2 \%$ \\
\hline 104 & University of Zurich (UZH) & Switzerland & 101.37 & 422 & 104.36 & $-2.9 \%$ \\
\hline 105 & National Institute for Material Science (NIMS) & Japan & 100.71 & 240 & 81.79 & $23.1 \%$ \\
\hline 106 & Pierre and Marie Curie University (UPMC) & France & 100.44 & 1,372 & 96.46 & $4.1 \%$ \\
\hline 107 & Agency for Science, Technology and Research (A*STAR) & Singapore & 100.33 & 284 & 81.62 & $22.9 \%$ \\
\hline 108 & Hokkaido University (HU) & Japan & 100.03 & 234 & 105.93 & $-5.6 \%$ \\
\hline 109 & Utrecht University (UU) & Netherlands & 99.89 & 475 & 111.50 & $-10.4 \%$ \\
\hline 110 & Kyushu University & Japan & 98.31 & 275 & 84.56 & $16.3 \%$ \\
\hline 111 & Rice University & United States & 97.96 & 275 & 82.53 & $18.7 \%$ \\
\hline 112 & University of Alberta ( $U$ of $A$ ) & Canada & 97.78 & 295 & 101.74 & $-3.9 \%$ \\
\hline 113 & University of Göttingen & Germany & 97.69 & 369 & 98.58 & $-0.9 \%$ \\
\hline 114 & Hebrew University of Jerusalem (HUJI) & Israel & 95.18 & 245 & 91.30 & $4.3 \%$ \\
\hline 115 & University of California Riverside (UCR) & United States & 94.82 & 279 & 77.71 & $22.0 \%$ \\
\hline 116 & University of Groningen (RUG) & Netherlands & 94.80 & 425 & 74.72 & $26.9 \%$ \\
\hline 117 & University of Würzburg & Germany & 93.86 & 260 & 95.03 & $-1.2 \%$ \\
\hline 118 & Indian Institute of Science (IISC) & India & 93.72 & 149 & 83.64 & $12.0 \%$ \\
\hline 119 & Nankai University (NKU) & China & 93.09 & 222 & 113.52 & $-18.0 \%$ \\
\hline 120 & Xiamen University (XMU) & China & 92.73 & 204 & 76.02 & $22.0 \%$ \\
\hline 121 & National Institute of Standards and Technology (NIST) & United States & 92.54 & 329 & 88.53 & $4.5 \%$ \\
\hline 122 & University of Rochester (UR) & United States & 92.34 & 291 & 104.73 & $-11.8 \%$ \\
\hline 123 & Boston University (BU) & United States & 92.14 & 389 & 99.12 & $-7.0 \%$ \\
\hline 124 & University of Basel & Switzerland & 90.89 & 251 & 87.67 & $3.7 \%$ \\
\hline 125 & Wuhan University (WHU) & China & 90.67 & 154 & 98.80 & $-8.2 \%$ \\
\hline 126 & Sichuan University (SCU) & China & 89.44 & 167 & 76.83 & $16.4 \%$ \\
\hline 127 & University of Leuven (KU Leuven) & Belgium & 88.93 & 393 & 91.46 & $-2.8 \%$ \\
\hline 128 & National Aeronautics and Space Administration (NASA) & United States & 88.79 & 1,006 & 94.01 & $-5.6 \%$ \\
\hline 129 & Memorial Sloan-Kettering Cancer Center (MSKCC) & United States & 88.29 & 217 & 75.15 & $17.5 \%$ \\
\hline 130 & University of New South Wales (UNSW) & Australia & 88.11 & 277 & 69.81 & $26.2 \%$ \\
\hline 131 & University of Chinese Academy of Sciences (UCAS) & China & 88.05 & 516 & 71.24 & $23.6 \%$ \\
\hline 132 & Sun Yat-sen University (SYSU) & China & 87.87 & 191 & 79.43 & $10.6 \%$ \\
\hline 133 & The University of Arizona (UA) & United States & 87.57 & 522 & 78.02 & $12.2 \%$ \\
\hline 134 & Australian National University (ANU) & Australia & 87.16 & 498 & 93.53 & $-6.8 \%$ \\
\hline 135 & Tel Aviv University (TAU) & Israel & 86.99 & 318 & 78.16 & $11.3 \%$ \\
\hline 136 & Soochow University & China & 86.12 & 158 & 65.31 & $31.9 \%$ \\
\hline 137 & Baylor College of Medicine (BCM) & United States & 85.63 & 213 & 76.15 & $12.5 \%$ \\
\hline 138 & Aarhus University (AU) & Denmark & 85.28 & 294 & 77.35 & $10.3 \%$ \\
\hline 139 & University of Erlangen-Nuremberg (FAU) & Germany & 84.46 & 248 & 84.55 & $-0.1 \%$ \\
\hline 140 & Oak Ridge National Laboratory (ORNL) & United States & 84.36 & 269 & 86.77 & $-2.8 \%$ \\
\hline 141 & Brown University & United States & 83.87 & 245 & 71.51 & $17.3 \%$ \\
\hline 142 & Humboldt University of Berlin (HU Berlin) & Germany & 83.71 & 356 & 76.98 & $8.7 \%$ \\
\hline 143 & The University of Melbourne (UniMelb) & Australia & 82.60 & 478 & 89.76 & $-8.0 \%$ \\
\hline 144 & Academia Sinica & Taiwan & 81.82 & 476 & 80.21 & $2.0 \%$ \\
\hline 145 & U.S. Department of Energy (DOE) & United States & 81.44 & 173 & 74.77 & $8.9 \%$ \\
\hline 146 & The University of Virginia (UVA) & United States & 81.39 & 319 & 77.87 & $4.5 \%$ \\
\hline 147 & Lund University (LU) & Sweden & 81.10 & 328 & 72.99 & $11.1 \%$ \\
\hline 148 & East China Normal University (ECNU) & China & 80.63 & 144 & 65.56 & $23.0 \%$ \\
\hline 149 & National Institute for Health and Medical Research (INSERM) & France & 80.44 & 654 & 70.08 & $14.8 \%$ \\
\hline 150 & University of Bonn (Uni Bonn) & Germany & 80.37 & 464 & 78.01 & $3.0 \%$ \\
\hline
\end{tabular}




\begin{tabular}{|c|c|c|c|c|c|c|}
\hline 2014 & INSTITUTION & COUNTRY & WFC & $\begin{array}{l}\text { ARTICLE } \\
\text { COUNT }\end{array}$ & $\begin{array}{l}2013 \\
\text { WFC }\end{array}$ & $\begin{array}{l}\text { 2013-2014 } \\
\text { CHANGE IN WFC }\end{array}$ \\
\hline 151 & Arizona State University (ASU) & United States & 79.94 & 255 & 80.58 & $-0.8 \%$ \\
\hline 152 & The University of Sydney (USYD) & Australia & 79.89 & 492 & 95.14 & $-16.0 \%$ \\
\hline 153 & University of Southampton (Soton) & United Kingdom & 79.04 & 315 & 85.53 & $-7.6 \%$ \\
\hline 154 & Technion-Israel Institute of Technology (IIT) & Israel & 78.91 & 231 & 67.90 & $16.2 \%$ \\
\hline 155 & University of Montreal & Canada & 78.01 & 327 & 68.73 & $13.5 \%$ \\
\hline 156 & Stony Brook University (SBU) & United States & 77.80 & 302 & 78.79 & $-1.3 \%$ \\
\hline 157 & Brookhaven National Laboratory (BNL) & United States & 77.63 & 323 & 67.83 & $14.4 \%$ \\
\hline 158 & University of Freiburg & Germany & 77.39 & 282 & 72.75 & $6.4 \%$ \\
\hline 159 & Uppsala University (UU) & Sweden & 76.75 & 351 & 80.05 & $-4.1 \%$ \\
\hline 160 & Durham University & United Kingdom & 76.61 & 386 & 84.05 & $-8.8 \%$ \\
\hline 161 & The University of Nottingham (UoN) & United Kingdom & 76.54 & 236 & 74.17 & $3.2 \%$ \\
\hline 162 & University of Hamburg (UHH) & Germany & 76.48 & 361 & 83.82 & $-8.8 \%$ \\
\hline 163 & Indian Institute of Science Education and Research (IISER) & India & 75.88 & 106 & 58.72 & $29.2 \%$ \\
\hline 164 & RWTH Aachen University (RWTH Aachen) & Germany & 75.57 & 255 & 87.18 & $-13.3 \%$ \\
\hline 165 & Case Western Reserve University (CWRU) & United States & 75.33 & 201 & 76.24 & $-1.2 \%$ \\
\hline 166 & Joseph Fourier University (UJF) & France & 74.84 & 682 & 69.43 & $7.8 \%$ \\
\hline 167 & Leiden University & Netherlands & 73.69 & 482 & 73.08 & $0.8 \%$ \\
\hline 168 & Hong Kong University of Science and Technology (HKUST) & China & 73.59 & 133 & 54.60 & $34.8 \%$ \\
\hline 169 & Hunan University (HNU) & China & 73.23 & 105 & 54.57 & $34.2 \%$ \\
\hline 170 & lowa State University (ISU) & United States & 72.56 & 208 & 78.91 & $-8.0 \%$ \\
\hline 171 & Medical Research Council (MRC) & United Kingdom & 71.76 & 470 & 67.17 & $6.8 \%$ \\
\hline 172 & The University of Hong Kong (HKU) & China & 71.75 & 185 & 71.38 & $0.5 \%$ \\
\hline 173 & Radboud University Nijmegen (RU) & Netherlands & 71.73 & 461 & 63.38 & $13.2 \%$ \\
\hline 174 & University of Waterloo & Canada & 71.02 & 199 & 61.22 & $16.0 \%$ \\
\hline 175 & Yonsei University & South Korea & 71.02 & 201 & 61.62 & $15.3 \%$ \\
\hline 176 & Karolinska Institute (KI) & Sweden & 70.88 & 234 & 59.23 & $19.7 \%$ \\
\hline 177 & University of Massachusetts Medical School (UMMS) & United States & 70.59 & 143 & 72.00 & $-2.0 \%$ \\
\hline 178 & Pohang University of Science and Technology (POSTECH) & South Korea & 70.38 & 151 & 70.00 & $0.5 \%$ \\
\hline 179 & University of Notre Dame & United States & 70.37 & 258 & 86.33 & $-18.5 \%$ \\
\hline 180 & University of Cologne (UoC) & Germany & 69.90 & 235 & 57.15 & $22.3 \%$ \\
\hline 181 & University of California Santa Cruz (UCSC) & United States & 69.85 & 537 & 58.03 & $20.4 \%$ \\
\hline 182 & National Institute of Advanced Industrial Science and Technology (AIST) & Japan & 69.79 & 185 & 79.48 & $-12.2 \%$ \\
\hline 183 & University of Warwick (Warwick) & United Kingdom & 69.68 & 392 & 61.07 & $14.1 \%$ \\
\hline 184 & King's College London (KCL) & United Kingdom & 69.57 & 239 & 71.59 & $-2.8 \%$ \\
\hline 185 & The Rockefeller University & United States & 69.55 & 207 & 82.62 & $-15.8 \%$ \\
\hline 186 & University of Barcelona (UB) & Spain & 68.98 & 608 & 72.14 & $-4.4 \%$ \\
\hline 187 & Xi'an Jiaotong University (XJTU) & China & 67.98 & 167 & 42.98 & $58.2 \%$ \\
\hline 188 & Stockholm University & Sweden & 67.67 & 416 & 71.69 & $-5.6 \%$ \\
\hline 189 & East China University of Science and Technology (ECUST) & China & 67.48 & 119 & 56.75 & $18.9 \%$ \\
\hline 190 & University of Sheffield & United Kingdom & 66.68 & 266 & 67.59 & $-1.3 \%$ \\
\hline 191 & University of Tübingen & Germany & 66.65 & 279 & 77.43 & $-13.9 \%$ \\
\hline 192 & University of Amsterdam (UvA) & Netherlands & 66.56 & 532 & 60.41 & $10.2 \%$ \\
\hline 193 & University of Paris Sud (Paris 11) & France & 65.88 & 611 & 72.33 & $-8.9 \%$ \\
\hline 194 & University of Glasgow & United Kingdom & 65.30 & 539 & 66.48 & $-1.8 \%$ \\
\hline 195 & National Tsing Hua University (NTHU) & Taiwan & 65.28 & 171 & 66.23 & $-1.4 \%$ \\
\hline 196 & University of Ottawa (uOttawa) & Canada & 64.43 & 147 & 59.20 & $8.8 \%$ \\
\hline 197 & The University of Texas MD Anderson Cancer Center & United States & 64.21 & 178 & 65.99 & $-2.7 \%$ \\
\hline 198 & Icahn School of Medicine at Mount Sinai (ISMMS) & United States & 64.14 & 200 & 53.91 & $19.0 \%$ \\
\hline 199 & University of Regensburg (UR) & Germany & 63.91 & 131 & 53.73 & $18.9 \%$ \\
\hline 200 & Technische Universität Berlin (TU Berlin) & Germany & 63.52 & 141 & 55.40 & $14.7 \%$ \\
\hline
\end{tabular}




\section{TOP INSTITUTIONS: LIFE SCIENCES}

\begin{tabular}{|c|c|c|c|c|c|c|}
\hline 2014 & INSTITUTION & COUNTRY & WFC & $\begin{array}{l}\text { ARTICLEE } \\
\text { COUNT }\end{array}$ & $\begin{array}{l}2013 \\
\text { WFC }\end{array}$ & $\begin{array}{l}2013-2014 \\
\text { CHANGE IN WFC }\end{array}$ \\
\hline 1 & Harvard University & United States & 610.16 & 1,363 & 587.65 & $3.8 \%$ \\
\hline 2 & National Institutes of Health $(\mathrm{NIH})$ & United States & 320.93 & 728 & 369.99 & $-13.3 \%$ \\
\hline 3 & Stanford University (SU) & United States & 226.07 & 465 & 245.14 & $-7.8 \%$ \\
\hline 4 & Max Planck Society & Germany & 225.42 & 707 & 216.73 & $4.0 \%$ \\
\hline 5 & Massachusetts Institute of Technology (MIT) & United States & 182.86 & 578 & 183.17 & $-0.2 \%$ \\
\hline 6 & Yale University & United States & 179.07 & 400 & 189.31 & $-5.4 \%$ \\
\hline 7 & University of California San Francisco (UCSF) & United States & 177.02 & 418 & 211.77 & $-16.4 \%$ \\
\hline 8 & University of California, San Diego (UC San Diego) & United States & 168.43 & 390 & 182.69 & $-7.8 \%$ \\
\hline 9 & University of Toronto ( $\mathrm{U}$ of $\mathrm{T}$ ) & Canada & 157.03 & 381 & 128.58 & $22.1 \%$ \\
\hline 10 & Chinese Academy of Sciences (CAS) & China & 155.22 & 420 & 147.94 & $4.9 \%$ \\
\hline 11 & French National Centre for Scientific Research (CNRS) & France & 155.06 & 972 & 145.94 & $6.2 \%$ \\
\hline 12 & University of Oxford & United Kingdom & 152.07 & 420 & 147.83 & $2.9 \%$ \\
\hline 13 & The Johns Hopkins University (JHU) & United States & 146.19 & 333 & 140.18 & $4.3 \%$ \\
\hline 14 & Columbia University in the City of New York (CU) & United States & 142.53 & 368 & 127.22 & $12.0 \%$ \\
\hline 15 & University of Pennsylvania (Penn) & United States & 141.62 & 320 & 175.58 & $-19.3 \%$ \\
\hline 16 & University of Michigan (U-M) & United States & 133.65 & 304 & 143.00 & $-6.5 \%$ \\
\hline 17 & University of Cambridge & United Kingdom & 133.57 & 399 & 155.77 & $-14.3 \%$ \\
\hline 18 & University of Washington (UW) & United States & 125.90 & 339 & 137.32 & $-8.3 \%$ \\
\hline 19 & University of California Los Angeles (UCLA) & United States & 123.06 & 313 & 126.98 & $-3.1 \%$ \\
\hline 20 & University of California Berkeley (UC Berkeley) & United States & 121.51 & 300 & 126.58 & $-4.0 \%$ \\
\hline 21 & Washington University in St. Louis (WUSTL) & United States & 121.40 & 279 & 137.34 & $-11.6 \%$ \\
\hline 22 & Cornell University & United States & 113.45 & 346 & 140.95 & $-19.5 \%$ \\
\hline 23 & Duke University & United States & 112.08 & 320 & 113.86 & $-1.6 \%$ \\
\hline 24 & University College London (UCL) & United Kingdom & 106.93 & 331 & 115.85 & $-7.7 \%$ \\
\hline 25 & The University of Texas Southwestern Medical Center (UT Southwestern Medical Center) & United States & 104.98 & 211 & 107.98 & $-2.8 \%$ \\
\hline 26 & The University of Tokyo (UTokyo) & Japan & 99.33 & 262 & 112.60 & $-11.8 \%$ \\
\hline 27 & New York University (NYU) & United States & 99.21 & 238 & 92.73 & $7.0 \%$ \\
\hline 28 & University of North Carolina at Chapel Hill (UNC) & United States & 97.89 & 240 & 93.85 & $4.3 \%$ \\
\hline 29 & The University of Chicago (UChicago) & United States & 94.05 & 234 & 93.92 & $0.1 \%$ \\
\hline 30 & University of Wisconsin-Madison (UW Madison) & United States & 92.41 & 182 & 87.38 & $5.8 \%$ \\
\hline 31 & Helmholtz Association of German Research Centres (Helmholtz) & Germany & 90.06 & 432 & 85.65 & $5.2 \%$ \\
\hline 32 & Baylor College of Medicine (BCM) & United States & 84.40 & 210 & 74.30 & $13.6 \%$ \\
\hline 33 & Vanderbilt University (VU) & United States & 80.27 & 196 & 74.80 & $7.3 \%$ \\
\hline 34 & University of California Davis (UC Davis) & United States & 79.61 & 194 & 90.61 & $-12.1 \%$ \\
\hline 35 & Memorial Sloan-Kettering Cancer Center (MSKCC) & United States & 77.02 & 194 & 68.47 & $12.5 \%$ \\
\hline 36 & Swiss Federal Institute of Technology Zurich (ETH Zurich) & Switzerland & 76.08 & 207 & 46.48 & $63.7 \%$ \\
\hline 37 & Northwestern University (NU) & United States & 75.07 & 172 & 91.03 & $-17.5 \%$ \\
\hline 38 & Emory University & United States & 74.39 & 181 & 66.89 & $11.2 \%$ \\
\hline 39 & University of Pittsburgh (Pitt) & United States & 73.58 & 180 & 81.12 & $-9.3 \%$ \\
\hline 40 & National Institute for Health and Medical Research (INSERM) & France & 71.33 & 575 & 62.47 & $14.2 \%$ \\
\hline 41 & The Scripps Research Institute (TSRI) & United States & 71.09 & 193 & 95.51 & $-25.6 \%$ \\
\hline 42 & McGill University & Canada & 71.03 & 208 & 78.78 & $-9.8 \%$ \\
\hline 43 & Rutgers, The State University of New Jersey (RU) & United States & 71.00 & 163 & 61.90 & $14.7 \%$ \\
\hline 44 & University of Massachusetts Medical School (UMMS) & United States & 66.97 & 137 & 69.40 & $-3.5 \%$ \\
\hline 45 & Spanish National Research Council (CSIC) & Spain & 66.91 & 295 & 62.94 & $6.3 \%$ \\
\hline 46 & The Rockefeller University & United States & 66.68 & 146 & 77.26 & $-13.7 \%$ \\
\hline 47 & Karolinska Institute (KI) & Sweden & 65.96 & 222 & 55.50 & $18.8 \%$ \\
\hline 48 & Imperial College London (ICL) & United Kingdom & 65.08 & 244 & 54.58 & $19.2 \%$ \\
\hline 49 & The University of British Columbia (UBC) & Canada & 64.55 & 175 & 53.90 & $19.8 \%$ \\
\hline 50 & Icahn School of Medicine at Mount Sinai (ISMMS) & United States & 63.95 & 198 & 52.10 & $22.8 \%$ \\
\hline
\end{tabular}


TOP INSTITUTIONS: EARTH \& ENVIRONMENTAL SCIENCES

\begin{tabular}{|c|c|c|c|c|c|c|}
\hline 2014 & INSTITUTION & COUNTRY & WFC & $\begin{array}{l}\text { ARTICLE } \\
\text { COUNT }\end{array}$ & $\begin{array}{l}2013 \\
\mathrm{WFC}\end{array}$ & $\begin{array}{c}2013-2014 \\
\text { CHANGE IN WFC }\end{array}$ \\
\hline 1 & Chinese Academy of Sciences (CAS) & China & 74.88 & 192 & 77.12 & $-2.9 \%$ \\
\hline 2 & French National Centre for Scientific Research (CNRS) & France & 74.14 & 479 & 56.92 & $30.2 \%$ \\
\hline 3 & Helmholtz Association of German Research Centres (Helmholtz) & Germany & 68.96 & 205 & 63.81 & $8.1 \%$ \\
\hline 4 & University of California, San Diego (UC San Diego) & United States & 54.32 & 131 & 47.45 & $14.5 \%$ \\
\hline 5 & National Aeronautics and Space Administration (NASA) & United States & 52.68 & 200 & 57.95 & $-9.1 \%$ \\
\hline 6 & National Oceanic and Atmospheric Administration (NOAA) & United States & 50.32 & 165 & 59.91 & $-16.0 \%$ \\
\hline 7 & California Institute of Technology (Caltech) & United States & 50.17 & 156 & 50.91 & $-1.5 \%$ \\
\hline 8 & University of Washington (UW) & United States & 49.12 & 111 & 42.34 & $16.0 \%$ \\
\hline 9 & Swiss Federal Institute of Technology Zurich (ETH Zurich) & Switzerland & 41.69 & 119 & 33.17 & $25.7 \%$ \\
\hline 10 & Woods Hole Oceanographic Institution (WHOI) & United States & 41.31 & 107 & 31.00 & $33.3 \%$ \\
\hline 11 & U.S. Geological Survey (USGS) & United States & 38.62 & 104 & 39.76 & $-2.9 \%$ \\
\hline 12 & The University of Texas at Austin (UT Austin) & United States & 37.58 & 83 & 21.11 & $78.0 \%$ \\
\hline 13 & Massachusetts Institute of Technology (MIT) & United States & 37.09 & 100 & 20.62 & $79.8 \%$ \\
\hline 14 & University of Colorado Boulder (CU-Boulder) & United States & 36.78 & 132 & 44.08 & $-16.6 \%$ \\
\hline 15 & The University of Tokyo (UTokyo) & Japan & 36.65 & 85 & 37.33 & $-1.8 \%$ \\
\hline 16 & Columbia University in the City of New York (CU) & United States & 32.65 & 105 & 41.25 & $-20.8 \%$ \\
\hline 17 & Pennsylvania State University (Penn State) & United States & 32.14 & 66 & 20.15 & $59.5 \%$ \\
\hline 18 & University of California Berkeley (UC Berkeley) & United States & 30.95 & 91 & 22.97 & $34.8 \%$ \\
\hline 19 & University of Cambridge & United Kingdom & 28.08 & 75 & 18.75 & $49.7 \%$ \\
\hline 20 & National Center for Atmospheric Research (NCAR) & United States & 25.08 & 81 & 25.37 & $-1.2 \%$ \\
\hline 21 & Stanford University (SU) & United States & 24.65 & 64 & 32.09 & $-23.2 \%$ \\
\hline 22 & University of Hawaii at Manoa (UH Manoa) & United States & 23.86 & 75 & 28.04 & $-14.9 \%$ \\
\hline 23 & Utrecht University (UU) & Netherlands & 23.00 & 59 & 21.31 & $8.0 \%$ \\
\hline 24 & University of California Davis (UC Davis) & United States & 22.96 & 59 & 16.60 & $38.3 \%$ \\
\hline 25 & Japan Agency for Marine-Earth Science and Technology (JAMSTEC) & Japan & 22.61 & 71 & 28.62 & $-21.0 \%$ \\
\hline 26 & University of Maryland, College Park (UMCP) & United States & 22.51 & 86 & 15.96 & $41.1 \%$ \\
\hline 27 & Princeton University & United States & 22.41 & 72 & 20.74 & $8.1 \%$ \\
\hline 28 & Institute of Research for Development (IRD) & France & 22.18 & 265 & 16.82 & $31.8 \%$ \\
\hline 29 & Spanish National Research Council (CSIC) & Spain & 22.02 & 97 & 18.56 & $18.6 \%$ \\
\hline 30 & Yale University & United States & 21.88 & 52 & 17.51 & $25.0 \%$ \\
\hline 31 & Australian National University (ANU) & Australia & 20.98 & 70 & 18.55 & $13.1 \%$ \\
\hline 32 & University of Bristol (UoB) & United Kingdom & 20.51 & 57 & 17.84 & $15.0 \%$ \\
\hline 33 & University of Wisconsin-Madison (UW Madison) & United States & 20.22 & 64 & 24.37 & $-17.0 \%$ \\
\hline 34 & University of Oxford & United Kingdom & 20.05 & 77 & 33.87 & $-40.8 \%$ \\
\hline 35 & Harvard University & United States & 19.46 & 61 & 32.26 & $-39.7 \%$ \\
\hline 36 & University of California Los Angeles (UCLA) & United States & 19.05 & 54 & 20.81 & $-8.5 \%$ \\
\hline 37 & Max Planck Society & Germany & 18.91 & 86 & 23.81 & $-20.6 \%$ \\
\hline 38 & Oregon State University (OSU) & United States & 18.91 & 50 & 19.77 & $-4.4 \%$ \\
\hline 39 & The University of Arizona (UA) & United States & 18.19 & 56 & 15.43 & $17.9 \%$ \\
\hline 40 & University of Bremen & Germany & 18.17 & 52 & 9.65 & $88.4 \%$ \\
\hline 41 & University of Toronto ( $\mathrm{U}$ of $\mathrm{T})$ & Canada & 18.16 & 43 & 12.10 & $50.1 \%$ \\
\hline 42 & National Institute of Geophysics and Volcanology (INGV) & Italy & 17.95 & 41 & 18.73 & $-4.2 \%$ \\
\hline 43 & Brown University & United States & 17.95 & 43 & 8.59 & $108.9 \%$ \\
\hline 44 & University of Minnesota (UMN) & United States & 17.93 & 57 & 15.15 & $18.4 \%$ \\
\hline 45 & Colorado State University (CSU) & United States & 17.51 & 47 & 17.98 & $-2.6 \%$ \\
\hline 46 & The Commonwealth Scientific and Industrial Research Organisation (CSIRO) & Australia & 17.34 & 63 & 16.92 & $2.4 \%$ \\
\hline 47 & Paris Diderot University (Paris 7) & France & 17.07 & 83 & 8.17 & $108.9 \%$ \\
\hline 48 & University of Florida (UF) & United States & 16.88 & 44 & 16.78 & $0.6 \%$ \\
\hline 49 & University of Miami (UM) & United States & 16.25 & 35 & 10.15 & $60.1 \%$ \\
\hline 50 & U.S. Department of Energy (DOE) & United States & 16.04 & 47 & 16.44 & $-2.4 \%$ \\
\hline
\end{tabular}




\section{TOP INSTITUTIONS: CHEMISTRY}

\begin{tabular}{|c|c|c|c|c|c|c|}
\hline 2014 & INSTITUTION & COUNTRY & WFC & $\begin{array}{l}\text { ARTICLE } \\
\text { COUNT }\end{array}$ & $\begin{array}{l}2013 \\
\text { WFC }\end{array}$ & $\begin{array}{l}\text { 2013-2014 } \\
\text { CHANGE IN WFC }\end{array}$ \\
\hline 1 & Chinese Academy of Sciences (CAS) & China & 771.24 & 1,396 & 681.87 & $13.1 \%$ \\
\hline 2 & French National Centre for Scientific Research (CNRS) & France & 251.51 & 921 & 227.97 & $10.3 \%$ \\
\hline 3 & Max Planck Society & Germany & 184.21 & 425 & 202.85 & $-9.2 \%$ \\
\hline 4 & Massachusetts Institute of Technology (MIT) & United States & 169.74 & 274 & 164.75 & $3.0 \%$ \\
\hline 5 & Stanford University (SU) & United States & 159.72 & 254 & 145.69 & $9.6 \%$ \\
\hline 6 & Peking University (PKU) & China & 151.32 & 366 & 142.68 & $6.1 \%$ \\
\hline 7 & Kyoto University & Japan & 151.26 & 237 & 157.12 & $-3.7 \%$ \\
\hline 8 & The University of Tokyo (UTokyo) & Japan & 143.72 & 228 & 159.48 & $-9.9 \%$ \\
\hline 9 & Nanyang Technological University (NTU) & Singapore & 143.06 & 217 & 123.90 & $15.5 \%$ \\
\hline 10 & Harvard University & United States & 141.23 & 256 & 143.52 & $-1.6 \%$ \\
\hline 11 & University of California Berkeley (UC Berkeley) & United States & 140.86 & 289 & 131.56 & $7.1 \%$ \\
\hline 12 & Northwestern University (NU) & United States & 136.36 & 211 & 152.92 & $-10.8 \%$ \\
\hline 13 & Swiss Federal Institute of Technology Zurich (ETH Zurich) & Switzerland & 127.29 & 217 & 102.80 & $23.8 \%$ \\
\hline 14 & University of Oxford & United Kingdom & 126.10 & 202 & 119.39 & $5.6 \%$ \\
\hline 15 & Zhejiang University (ZJU) & China & 125.25 & 178 & 86.42 & $44.9 \%$ \\
\hline 16 & Nanjing University (NJU) & China & 120.32 & 207 & 117.34 & $2.5 \%$ \\
\hline 17 & Swiss Federal Institute of Technology in Lausanne (EPFL) & Switzerland & 116.01 & 219 & 80.35 & $44.4 \%$ \\
\hline 18 & Fudan University & China & 114.02 & 186 & 80.10 & $42.3 \%$ \\
\hline 19 & The University of Texas at Austin (UT Austin) & United States & 112.69 & 161 & 109.67 & $2.7 \%$ \\
\hline 20 & University of Cambridge & United Kingdom & 110.12 & 186 & 105.84 & $4.0 \%$ \\
\hline 21 & University of Illinois at Urbana Champaign (UIUC) & United States & 108.91 & 191 & 115.67 & $-5.8 \%$ \\
\hline 22 & Helmholtz Association of German Research Centres (Helmholtz) & Germany & 108.35 & 302 & 91.62 & $18.3 \%$ \\
\hline 23 & University of Science and Technology of China (USTC) & China & 108.19 & 223 & 93.78 & $15.4 \%$ \\
\hline 24 & Indian Institutes of Technology (IITs) & India & 106.58 & 141 & 74.77 & $42.6 \%$ \\
\hline 25 & Osaka University & Japan & 105.69 & 171 & 106.33 & $-0.6 \%$ \\
\hline 26 & Tsinghua University (TH) & China & 101.79 & 201 & 92.06 & $10.6 \%$ \\
\hline 27 & University of Wisconsin-Madison (UW Madison) & United States & 96.53 & 139 & 105.23 & $-8.3 \%$ \\
\hline 28 & National University of Singapore (NUS) & Singapore & 93.39 & 177 & 83.81 & $11.4 \%$ \\
\hline 29 & California Institute of Technology (Caltech) & United States & 92.35 & 144 & 107.31 & $-13.9 \%$ \\
\hline 30 & Council of Scientific and Industrial Research (CSIR) & India & 90.40 & 117 & 79.46 & $13.8 \%$ \\
\hline 31 & Lawrence Berkeley National Laboratory (LBNL) & United States & 85.03 & 248 & 74.57 & $14.0 \%$ \\
\hline 32 & Lanzhou University (LZU) & China & 84.31 & 118 & 50.35 & $67.5 \%$ \\
\hline 33 & University of California Santa Barbara (UCSB) & United States & 83.37 & 137 & 62.14 & $34.2 \%$ \\
\hline 34 & University of Pennsylvania (Penn) & United States & 82.19 & 124 & 80.23 & $2.4 \%$ \\
\hline 35 & Tohoku University & Japan & 81.32 & 138 & 62.89 & $29.3 \%$ \\
\hline 36 & The Scripps Research Institute (TSRI) & United States & 79.61 & 130 & 101.52 & $-21.6 \%$ \\
\hline 37 & University of California, San Diego (UC San Diego) & United States & 79.07 & 134 & 78.52 & $0.7 \%$ \\
\hline 38 & Jilin University (JLU) & China & 77.57 & 128 & 73.88 & $5.0 \%$ \\
\hline 39 & University of California Los Angeles (UCLA) & United States & 75.45 & 134 & 87.43 & $-13.7 \%$ \\
\hline 40 & University of North Carolina at Chapel Hill (UNC) & United States & 74.34 & 101 & 75.09 & $-1.0 \%$ \\
\hline 41 & The University of Manchester (UoM) & United Kingdom & 73.82 & 132 & 53.06 & $39.1 \%$ \\
\hline 42 & Imperial College London (ICL) & United Kingdom & 73.20 & 126 & 62.12 & $17.8 \%$ \\
\hline 43 & Korea Advanced Institute of Science and Technology (KAIST) & South Korea & 73.03 & 133 & 56.93 & $28.3 \%$ \\
\hline 44 & University of Michigan (U-M) & United States & 72.80 & 112 & 92.32 & $-21.1 \%$ \\
\hline 45 & Xiamen University (XMU) & China & 71.25 & 138 & 59.87 & $19.0 \%$ \\
\hline 46 & Ludwig Maximilian University of Munich (LMU) & Germany & 71.20 & 130 & 55.22 & $28.9 \%$ \\
\hline 47 & Yale University & United States & 71.10 & 122 & 46.80 & $51.9 \%$ \\
\hline 48 & Sichuan University (SCU) & China & 70.75 & 98 & 68.45 & $3.4 \%$ \\
\hline 49 & University of Toronto $(\mathrm{U}$ of $\mathrm{T})$ & Canada & 69.01 & 120 & 85.42 & $-19.2 \%$ \\
\hline 50 & Seoul National University (SNU) & South Korea & 68.64 & 111 & 70.70 & $-2.9 \%$ \\
\hline
\end{tabular}




\section{TOP INSTITUTIONS: PHYSICAL SCIENCES}

\begin{tabular}{|c|c|c|c|c|c|c|}
\hline 2014 & INSTITUTION & COUNTRY & WFC & $\begin{array}{l}\text { ARTICLE } \\
\text { COUNT }\end{array}$ & $\begin{array}{l}2013 \\
\text { WFC }\end{array}$ & $\begin{array}{l}2013-2014 \\
\text { CHANGE IN WFC }\end{array}$ \\
\hline 1 & Chinese Academy of Sciences (CAS) & China & 413.44 & 1,376 & 406.86 & $1.6 \%$ \\
\hline 2 & French National Centre for Scientific Research (CNRS) & France & 333.36 & 2,812 & 335.41 & $-0.6 \%$ \\
\hline 3 & Max Planck Society & Germany & 300.72 & 1,948 & 353.91 & $-15.0 \%$ \\
\hline 4 & Stanford University (SU) & United States & 221.86 & 652 & 211.18 & $5.1 \%$ \\
\hline 5 & Helmholtz Association of German Research Centres (Helmholtz) & Germany & 213.14 & 819 & 217.24 & $-1.9 \%$ \\
\hline 6 & Massachusetts Institute of Technology (MIT) & United States & 212.29 & 718 & 226.42 & $-6.2 \%$ \\
\hline 7 & The University of Tokyo (UTokyo) & Japan & 204.65 & 737 & 193.50 & $5.8 \%$ \\
\hline 8 & Harvard University & United States & 200.80 & 1,149 & 191.30 & $5.0 \%$ \\
\hline 9 & University of Cambridge & United Kingdom & 187.59 & 839 & 170.15 & $10.3 \%$ \\
\hline 10 & University of California Berkeley (UC Berkeley) & United States & 149.28 & 735 & 135.57 & $10.1 \%$ \\
\hline 11 & Swiss Federal Institute of Technology Zurich (ETH Zurich) & Switzerland & 140.09 & 520 & 132.35 & $5.8 \%$ \\
\hline 12 & University of California Santa Barbara (UCSB) & United States & 115.63 & 377 & 120.80 & $-4.3 \%$ \\
\hline 13 & University of Oxford & United Kingdom & 113.00 & 594 & 126.23 & $-10.5 \%$ \\
\hline 14 & University of Michigan (U-M) & United States & 112.36 & 464 & 121.38 & $-7.4 \%$ \\
\hline 15 & Peking University (PKU) & China & 111.97 & 454 & 105.09 & $6.6 \%$ \\
\hline 16 & Russian Academy of Sciences (RAS) & Russia & 111.96 & 724 & 109.38 & $2.4 \%$ \\
\hline 17 & Spanish National Research Council (CSIC) & Spain & 108.99 & 1,281 & 98.40 & $10.8 \%$ \\
\hline 18 & Swiss Federal Institute of Technology in Lausanne (EPFL) & Switzerland & 104.69 & 401 & 102.90 & $1.7 \%$ \\
\hline 19 & Princeton University & United States & 102.49 & 469 & 114.36 & $-10.4 \%$ \\
\hline 20 & The University of Texas at Austin (UT Austin) & United States & 98.37 & 330 & 98.67 & $-0.3 \%$ \\
\hline 21 & Tsinghua University $(\mathrm{TH})$ & China & 98.25 & 363 & 87.94 & $11.7 \%$ \\
\hline 22 & California Institute of Technology (Caltech) & United States & 95.56 & 839 & 100.29 & $-4.7 \%$ \\
\hline 23 & University of Maryland, College Park (UMCP) & United States & 90.92 & 542 & 96.06 & $-5.4 \%$ \\
\hline 24 & University of Illinois at Urbana-Champaign (UIUC) & United States & 86.99 & 297 & 111.83 & $-22.2 \%$ \\
\hline 25 & Lawrence Berkeley National Laboratory (LBNL) & United States & 86.75 & 514 & 76.52 & $13.4 \%$ \\
\hline 26 & University of California Los Angeles (UCLA) & United States & 81.40 & 314 & 97.72 & $-16.7 \%$ \\
\hline 27 & National University of Singapore (NUS) & Singapore & 80.80 & 171 & 88.74 & $-8.9 \%$ \\
\hline 28 & Kyoto University & Japan & 77.15 & 330 & 91.02 & $-15.2 \%$ \\
\hline 29 & Los Alamos National Laboratory (LANL) & United States & 76.30 & 289 & 84.24 & $-9.4 \%$ \\
\hline 30 & National Research Council (CNR) & Italy & 75.80 & 282 & 93.70 & $-19.1 \%$ \\
\hline 31 & Tohoku University & Japan & 75.65 & 211 & 96.92 & $-22.0 \%$ \\
\hline 32 & The University of Chicago (UChicago) & United States & 75.47 & 392 & 63.01 & $19.8 \%$ \\
\hline 33 & University of Science and Technology of China (USTC) & China & 74.91 & 301 & 71.40 & $4.9 \%$ \\
\hline 34 & University of California, San Diego (UC San Diego) & United States & 74.21 & 259 & 73.71 & $0.7 \%$ \\
\hline 35 & Nanyang Technological University (NTU) & Singapore & 72.88 & 141 & 71.87 & $1.4 \%$ \\
\hline 36 & Seoul National University (SNU) & South Korea & 72.34 & 242 & 66.08 & $9.5 \%$ \\
\hline 37 & Imperial College London (ICL) & United Kingdom & 71.34 & 370 & 79.35 & $-10.1 \%$ \\
\hline 38 & Northwestern University (NU) & United States & 71.33 & 239 & 91.07 & $-21.7 \%$ \\
\hline 39 & Pennsylvania State University (Penn State) & United States & 68.96 & 369 & 77.44 & $-10.9 \%$ \\
\hline 40 & Cornell University & United States & 68.66 & 265 & 73.02 & $-6.0 \%$ \\
\hline 41 & National Institute of Standards and Technology (NIST) & United States & 68.50 & 254 & 67.83 & $1.0 \%$ \\
\hline 42 & Nanjing University (NJU) & China & 68.43 & 233 & 64.94 & $5.4 \%$ \\
\hline 43 & National Institute for Material Science (NIMS) & Japan & 68.14 & 166 & 43.97 & $55.0 \%$ \\
\hline 44 & Osaka University & Japan & 67.71 & 245 & 62.61 & $8.1 \%$ \\
\hline 45 & Yale University & United States & 66.85 & 382 & 60.96 & $9.7 \%$ \\
\hline 46 & University of Colorado Boulder (CU-Boulder) & United States & 66.06 & 338 & 55.85 & $18.3 \%$ \\
\hline 47 & Atomic Energy and Alternative Energies Commission (CEA) & France & 65.66 & 765 & 91.23 & $-28.0 \%$ \\
\hline 48 & Argonne National Laboratory (ANL) & United States & 63.52 & 313 & 70.93 & $-10.4 \%$ \\
\hline 49 & Columbia University in the City of New York (CU) & United States & 61.89 & 351 & 74.99 & $-17.5 \%$ \\
\hline 50 & Korea Advanced Institute of Science and Technology (KAIST) & South Korea & 58.92 & 103 & 49.76 & $18.4 \%$ \\
\hline
\end{tabular}




\section{TOP INSTITUTIONS IN NATURE AND SCIENCE JOURNALS}

\begin{tabular}{|c|c|c|c|c|c|c|}
\hline 2014 & INSTITUTION & COUNTRY & WFC & $\begin{array}{l}\text { ARTICLE } \\
\text { COUNT }\end{array}$ & $\begin{array}{l}2013 \\
\text { WFC }\end{array}$ & $\begin{array}{l}\text { 2013-2014 } \\
\text { CHANGE IN WFC }\end{array}$ \\
\hline 1 & Harvard University & United States & 75.39 & 210 & 76.41 & $-1.3 \%$ \\
\hline 2 & Stanford University (SU) & United States & 41.44 & 103 & 38.30 & $8.2 \%$ \\
\hline 3 & Massachusetts Institute of Technology (MIT) & United States & 34.13 & 123 & 45.59 & $-25.1 \%$ \\
\hline 4 & Max Planck Society & Germany & 30.40 & 120 & 32.87 & $-7.5 \%$ \\
\hline 5 & National Institutes of Health $(\mathrm{NIH})$ & United States & 24.04 & 69 & 27.57 & $-12.8 \%$ \\
\hline 6 & University of California Berkeley (UC Berkeley) & United States & 21.35 & 95 & 21.58 & $-1.1 \%$ \\
\hline 7 & University of Washington (UW) & United States & 20.63 & 63 & 14.49 & $42.3 \%$ \\
\hline 8 & University of California, San Diego (UC San Diego) & United States & 20.29 & 54 & 17.83 & $13.9 \%$ \\
\hline 9 & Yale University & United States & 20.16 & 56 & 17.04 & $18.3 \%$ \\
\hline 10 & French National Centre for Scientific Research (CNRS) & France & 19.46 & 166 & 15.12 & $28.7 \%$ \\
\hline 11 & California Institute of Technology (Caltech) & United States & 18.61 & 62 & 12.73 & $46.2 \%$ \\
\hline 12 & University of Cambridge & United Kingdom & 18.12 & 82 & 20.69 & $-12.4 \%$ \\
\hline 13 & University of California San Francisco (UCSF) & United States & 17.96 & 48 & 24.04 & $-25.3 \%$ \\
\hline 14 & University of Oxford & United Kingdom & 17.79 & 60 & 13.84 & $28.5 \%$ \\
\hline 15 & Columbia University in the City of New York (CU) & United States & 17.45 & 64 & 19.75 & $-11.6 \%$ \\
\hline 16 & Helmholtz Association of German Research Centres (Helmholtz) & Germany & 16.66 & 80 & 10.95 & $52.1 \%$ \\
\hline 17 & The Scripps Research Institute (TSRI) & United States & 14.18 & 35 & 18.43 & $-23.1 \%$ \\
\hline 18 & The University of Tokyo (UTokyo) & Japan & 13.21 & 44 & 15.55 & $-15.1 \%$ \\
\hline 19 & Chinese Academy of Sciences (CAS) & China & 13.11 & 58 & 18.71 & $-29.9 \%$ \\
\hline 20 & University of Michigan (U-M) & United States & 13.04 & 44 & 9.36 & $39.3 \%$ \\
\hline 21 & Weizmann Institute of Science (WIS) & Israel & 12.91 & 22 & 6.67 & $93.5 \%$ \\
\hline 22 & Swiss Federal Institute of Technology Zurich (ETH Zurich) & Switzerland & 12.74 & 43 & 13.98 & $-8.9 \%$ \\
\hline 23 & Princeton University & United States & 12.29 & 32 & 13.50 & $-9.0 \%$ \\
\hline 24 & The University of Texas at Austin (UT Austin) & United States & 12.20 & 40 & 4.60 & $165.3 \%$ \\
\hline 25 & Cornell University & United States & 11.83 & 55 & 13.31 & $-11.1 \%$ \\
\hline 26 & The Johns Hopkins University (JHU) & United States & 11.82 & 39 & 12.33 & $-4.1 \%$ \\
\hline 27 & Washington University in St. Louis (WUSTL) & United States & 11.40 & 40 & 8.41 & $35.6 \%$ \\
\hline 28 & Medical Research Council (MRC) & United Kingdom & 11.34 & 24 & 9.29 & $22.0 \%$ \\
\hline 29 & University of Wisconsin-Madison (UW Madison) & United States & 11.14 & 35 & 3.16 & $252.1 \%$ \\
\hline 30 & Memorial Sloan-Kettering Cancer Center (MSKCC) & United States & 10.81 & 29 & 5.08 & $112.9 \%$ \\
\hline 31 & New York University (NYU) & United States & 10.54 & 25 & 10.55 & $-0.1 \%$ \\
\hline 32 & University of California Los Angeles (UCLA) & United States & 10.35 & 41 & 12.75 & $-18.8 \%$ \\
\hline 33 & The University of Texas Southwestern Medical Center (UT Southwestern Medical Center) & United States & 9.88 & 20 & 11.79 & $-16.2 \%$ \\
\hline 34 & The University of Chicago (UChicago) & United States & 9.33 & 47 & 11.75 & $-20.6 \%$ \\
\hline 35 & F. Hoffmann-La Roche Ltd & Switzerland & 9.18 & 20 & 2.58 & $255.9 \%$ \\
\hline 36 & The Rockefeller University & United States & 9.14 & 20 & 11.45 & $-20.2 \%$ \\
\hline 37 & University of Pennsylvania (Penn) & United States & 9.01 & 30 & 10.23 & $-11.9 \%$ \\
\hline 38 & Lawrence Berkeley National Laboratory (LBNL) & United States & 9.00 & 52 & 5.97 & $50.7 \%$ \\
\hline 39 & University of California Santa Cruz (UCSC) & United States & 8.93 & 37 & 4.54 & $96.6 \%$ \\
\hline 40 & University of Toronto ( $U$ of $T$ ) & Canada & 8.64 & 38 & 15.70 & $-44.9 \%$ \\
\hline 41 & Duke University & United States & 8.55 & 33 & 7.14 & $19.8 \%$ \\
\hline 42 & University of Maryland, College Park (UMCP) & United States & 8.49 & 40 & 5.21 & $63.1 \%$ \\
\hline 43 & Kyoto University & Japan & 7.44 & 25 & 7.48 & $-0.5 \%$ \\
\hline 44 & University of Copenhagen (UCPH) & Denmark & 7.43 & 50 & 5.11 & $45.6 \%$ \\
\hline 45 & RIKEN & Japan & 7.33 & 32 & 9.62 & $-23.8 \%$ \\
\hline 46 & University of California Davis (UC Davis) & United States & 7.13 & 29 & 8.06 & $-11.6 \%$ \\
\hline 47 & University College London (UCL) & United Kingdom & 7.10 & 41 & 7.69 & $-7.6 \%$ \\
\hline 48 & Pennsylvania State University (Penn State) & United States & 7.01 & 25 & 6.26 & $11.9 \%$ \\
\hline 49 & University of Zurich (UZH) & Switzerland & 6.70 & 19 & 4.81 & $39.1 \%$ \\
\hline 50 & Peking University (PKU) & China & 6.48 & 28 & 4.10 & $58.0 \%$ \\
\hline
\end{tabular}

Weighted fractional count (WFC) for each institution is shown to two decimal places only. When two or more institutions have the same WFC, their positions are determined by the thousandth place (or beyond).

These results are based on the most recent data available as of 11 September 2014 Owing to continual refinements of the data, the figures in the database are liable to change and might differ to those printed in the supplements. 